\title{
Pyrazines - valuable flavour \& fragrance compounds: Biocatalytic synthesis and industrial applications.
}

\author{
Frederik Mortzfeld ${ }^{1}$, Chiam Hashem ${ }^{2}$, Kvetka Vrankova ${ }^{3}$, Margit Winkler ${ }^{4}$, and Florian \\ Rudroff $^{1}$ \\ ${ }^{1}$ TU Wien Faculty of Technical Chemistry \\ ${ }^{2}$ Graz University of Technology \\ ${ }^{3}$ Axxence Slovakia, s.r.o. \\ ${ }^{4} \mathrm{ACIB} \mathrm{GmbH}$
}

April 28, 2020

\begin{abstract}
Substituted alkyl pyrazines - other than being extracted from various natural sources such as coffee beans, cocoa beans, nuts and vegetables - can be synthesized by the use of traditional chemical methods or by the help of certain microorganisms. The importance of pyrazines for food industry is expected to grow in the upcoming years due to the higher demand for ready meals, coffee and chocolate drinks; the roasty, nutty and earthy smell is reminiscent for coffee and cocoa depending on substitution and concentration of pyrazines. The growing awareness of people about the ingredients and the origin of their daily food has strongly influenced the market with labels like 'organic' and 'natural'. Many flavor ingredients prepared by biotechnology methods have conquered the market in recent years and are destined to replace and optimize the ineffective $(0.01 \%$ pyrazine $/ \mathrm{kg}$ biomass) extraction from plants or animal sources. This overview focuses on the achievements and the upcoming challenges in pyrazine synthesis. Major parts deal with the extraction of natural products from sugar molasses, the chemical synthesis, fermentation by microorganisms and preparative methods by biocatalysis. The different types of production are decisive for the declaration and value of the final product and can span from 200-3500 $\$ / \mathrm{kg}$ for the synthetically produced or the naturally extracted 2,5-dimethylpyrazine, respectively.
\end{abstract}

Mini-review / Perspective

Title: Pyrazines: Valuable flavor and fragrance compounds - Synthesis and industrial applications

[Frederik B. Mortzfeld] $]^{1}$,

[Chiam Hashem $]^{2}$

[Kvetoslava Vranková $]^{3}$

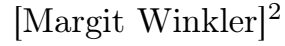

[Florian Rudroff] $]^{1, *}$

${ }^{1}$ Institute of Applied Synthetic Chemistry, TU Wien, Getreidemarkt 9, 1060 Vienna, Austria.

${ }^{2}$ Institute of Molecular Biotechnology, TU Graz, Petersgasse 12, 8010 Graz, Austria

${ }^{3}$ Axxence Slovakia, s.r.o., Mickiewiczova 9, 81107 Bratislava, Slovakia 
Correspondence: [Prof. Florian Rudroff, Institute of Applied Synthetic Chemistry, TU Wien, Getreidemarkt 9/163-OC, 1060 Vienna, Austria].

E-mail : [florian.rudroff@tuwien.ac.at]

Keywords: Biocatalysis, Biotransformation, Chemo-Enzymatic Reactions, Fragrance and Flavor Compounds, Pyrazine,

\title{
Abbreviations:
}

AmDH , amine dehydrogenase; AS , Acetolactate synthase;CAGR , Compound Annual Growth Rate; CDH , cellobiose dehydrogenase; GRAS , Generally recognized as safe;ox., oxidation; PDC, pyruvate decarboxylase;TDH, l-threonine-3-dehydrogenase; TDA,l-threonine deaminase; $t$-BuOK , Potassiumtert -butoxide; ThDP , thiamine diphosphate;SucA , ThDP-dependent E1 subunit of the $\alpha$-ketoglutarate dehydrogenase complex

\begin{abstract}
Substituted alkyl pyrazines - other than being extracted from various natural sources such as coffee beans, cocoa beans, nuts and vegetables - can be synthesized by the use of traditional chemical methods or by the help of certain microorganisms. The importance of pyrazines for food industry is expected to grow in the upcoming years due to the higher demand for ready meals, coffee and chocolate drinks; the roasty, nutty and earthy smell is reminiscent for coffee and cocoa depending on substitution and concentration of pyrazines. The growing awareness of people about the ingredients and the origin of their daily food has strongly influenced the market with labels like 'organic' and 'natural'. Many flavor ingredients prepared by biotechnology methods have conquered the market in recent years and are destined to replace and optimize the ineffective (0.01\% pyrazine/ $\mathrm{kg}$ biomass) extraction from plants or animal sources. This overview focuses on the achievements and the upcoming challenges in pyrazine synthesis. Major parts deal with the extraction of natural products from sugar molasses, the chemical synthesis, fermentation by microorganisms and preparative methods by biocatalysis. The different types of production are decisive for the declaration and value of the final product and can span from $200-3500 \$ / \mathrm{kg}$ for the synthetically produced or the naturally extracted 2,5-dimethylpyrazine, respectively.
\end{abstract}

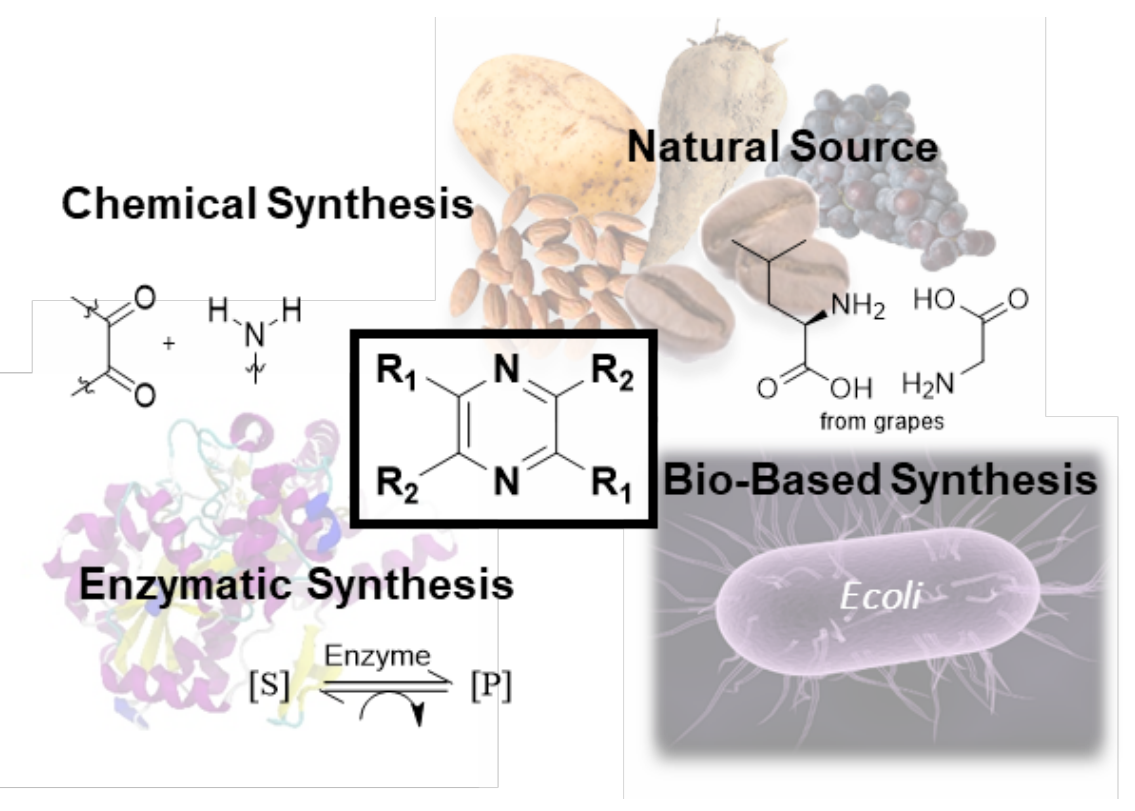

Graphical abstract. 


\section{Introduction}

\section{Short History $\&$ Appearances of Pyrazines, Extraction from Molasses}

Humankind has always been fascinated by the food it eats. Historically, herbs and spices were used to improve the taste and preserve the shelf life of food [1]. Later, thermal processing such as frying, cooking and baking marked the beginning of changing the properties and flavors of foods, indifferent about the occurrence of triggered organic reactions at high temperatures. Both, the advent of modern organic chemistry and the increasing demand of food flavors - from strawberry to vanilla - are the reasons why chemically synthesized flavors have appeared in our food. Many of these have been inspired by naturally occurring fermentation processes with the help of further achievements in chemistry, microbiology, biochemistry and genetics [2]. To cover global demands, further progress is necessary. For example, the world's strawberry harvest can cover only a small amount for strawberry flavored products such as strawberry jams, dairy products, ice cream, shampoos, room fragrances etc . and is often enriched in flavour with chemical or biological synthesized flavorings [personal consultations with Axxence Slovakia s.r.o.].

Today, most flavorings are produced by chemical synthesis or derived from natural sources. However, market analysis shows a customer trend craving products that are "natural' or at least containing "natural flavor" which means that ingredients are coming from natural sources such as a spice, fruit, or vegetable (see part of the EU regulation in chapter 2.4 (EEC No 1334/2008)). The legislation of the regulations varies from country to country. Most commercial flavor compounds do not qualify to be labelled 'natural' despite being the structural equivalent of natural flavors because they are synthesized [3] chemically. Biological preparation is more often hindered due to high production costs of compatible unit operations or deficiency in availability of sources. Vanilla flavor is a popular example since only less than $1 \%$ of its global consumption derives from vanilla orchids [4].\{Kundu, 2017 \#420;Fache, 2016 \#424\} Thousands of tons of vanillin are produced chemically, although 'biovanillin' is emerging [5]. Public perception increases the pressure on industrial processes towards more sustainability. However, extraction from plants is often limited by low concentrations of the desired compounds and their appearance in variable and complex mixtures.

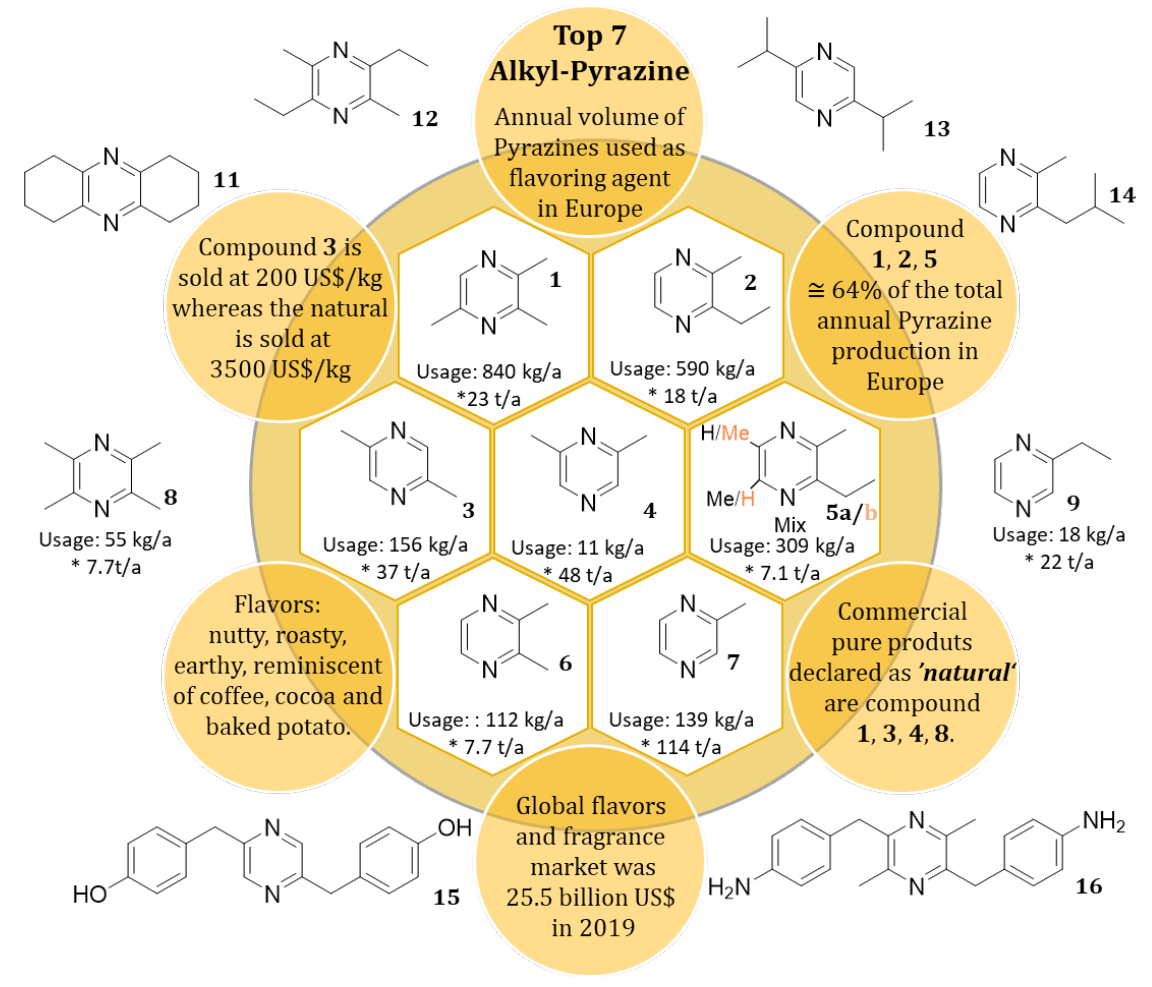


Figure 1. Overview on alkyl pyrazines. The most processed alkyl pyrazines1 - 7. Annual volumes of use of pyrazine derivatives used as flavoring agents in Europe 2004 is stated in $\mathrm{kg} /$ year, whereas the * represents the quantitative natural occurrence of alkyl pyrazines in foods in Europe in tonnes per year [6]. Special attention belongs to compounds $\mathbf{1}, \mathbf{3}, \mathbf{4}$, and $\mathbf{8}$ which are commercially available as pure substance declared as natural whereas others are at least partially chemical synthesized. Short notice: To the best of our knowledge these are the latest official volumes of the WHO and EFSA [7]. Non-commercial Synthetic alkyl-pyrazines11 -16 .

The consequences are high extraction costs and a product line that suffers from high risk of change in taste and smell. Therefore, industrial research is turning towards bio-based and/or biocatalytic strategies. Nowadays, the use of certain microorganisms [8] is well known and declared as natural and safe by food authorities [9] (EFSA, FDA etc. ), and offers the chance for a better economic feasibility with mild reaction conditions, high specificity and simplified downstream processes due to higher purities, better reproducibility and constant sourcing in contrast e.g. to the natural feedstock extraction.

Pyrazines are volatile, nitrogen-containing heterocyclic compounds widely distributed in plants [10], insects [11], fungi and bacteria [12]. They act as an odor signal to repel predators and effectively prevent vegetative tissue or immature fruit from being eaten [13]. For this reason, pyrazines find various applications as ingredients in pesticides, insecticides, dyes, and pharmaceutical compounds [14]. Pyrazines gained attention from the food industry as important ingredients in raw and roasted foods. Especially alkylated pyrazines are in the focus, as they have strong olfactory properties (Figure 1). They can be extracted from natural sources such as potatoes, nuts and coffee with applications in numerous areas, e.g. 2,3-dimethypyrazine 6 (coffee, nutty, roasty) is used in various beverages and sauces, while 2,5-dimethylpyrazine $\mathbf{3}$ (nutty, musty, earthy, roasted cocoa) is used in breakfast cereals. 2,3,5-Trimethylpyrazine $\mathbf{1}$ (chocolate enhancer) and 2,6-dimethylpyrazine 4 (roasty, nutty, beef) are used in meals with coffee, cocoa, meat or potatoes as a roasted flavour. 2-Ethyl-3-methylpyrazine $\mathbf{2}$ (nutty, musty, corn, raw, earthy, oily) is used in peanut products, popcorn, and in bread. The perceived odor depends on the dilution (recommended $0.1 \%$ or less in dipropylene glycol) of the substance. Methoxylated pyrazines are mainly used in the perfume industry to improve the odor of cosmetics and toiletries [15] (fresh, green, woody, ambery, oriental, musky, minty and herbaceous). Pyrazine flavors are often added to microwave foods because roasting odors are not formed in microwaves at temperatures below $200^{\circ} \mathrm{C}[16]$.

The growing demand for the 'natural' alkyl pyrazines described above cannot be satisfied by extraction and concentration techniques from natural sources alone, since the natural content of e.g. 0.01 wt. \% pyrazines in sugar beet molasses is low [14c, 17]. Modern biotechnology, recognized as a sustainable economic process, could cope with the growing demand for "natural' compounds in the future and may further increase the amount may fulfil the market demand of selectively substituted pyrazines.

\section{Different approaches to produce the same molecule}

Pyrazines are formed by the condensation of two molecules of $\alpha$-aminocarbonyls such as amino acids or amino sugars (Maillard reaction) [18]. The two aminocarbonyl compounds firstly react to form a dihydropyrazine and subsequently undergo aromatization by oxidation with air or elimination of a hydroxyl group to form the side chain. Pyrazines can be extracted and distilled in low amounts directly from natural sources such as vegetables, coffee, cocoa and nuts, most of them deliberately formed at elevated temperature in roasting processes in the case of coffee and cocoa $[17,19]$ (Figure 3, C ) or unintentionally in refining processes of e.g. white sugar [20]. 


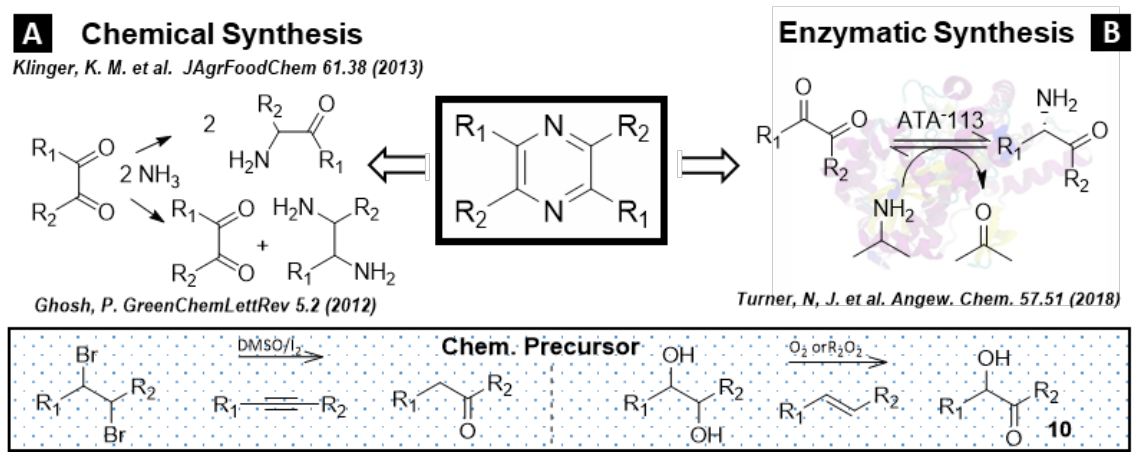

Figure 2. Retrosynthetic analysis shows different ways leading to the same molecule. Overview about different strategies: A) Chemical synthesis via amination of 1,2-diketones and subsequent dimerization/condensation to pyrazine starting from oxidized chemical precursors. B) Combination of chemical precursor and enzymatic amination synthesis using aminotransferase (ATA-113).

An example that has been studied over centuries is 3-isobutyl-2-methoxypyrazine, a characteristic odor in grapes (Figure 3,C ), which is biosynthesized by the plant from leucine and glycine [21] or leucinamide and glyoxal [10]. Efforts have been made to identify these hypothetical precursors without final confirmation.

In general, chemical synthesis cannot convert two amino acids to a pyrazine core without a suitable catalyst. However, it is feasible to start from 1,2-diketones and diamines or aminoketones and amino aldehydes or other synthetic equivalents (Figure 2, A ). The enzymatic synthesis can combine chemical precursors and enzyme catalysis to obtain the final product (Figure 2, B ). Microbial synthesis uses the cellular network of naturally pyrazine producing microorganisms to form selectively substituted pyrazines by feeding the microorganism the necessary precursors (Figure 3, D ). The advantages and challenges of chemistry, biocatalysis and biotechnology are summarized below.

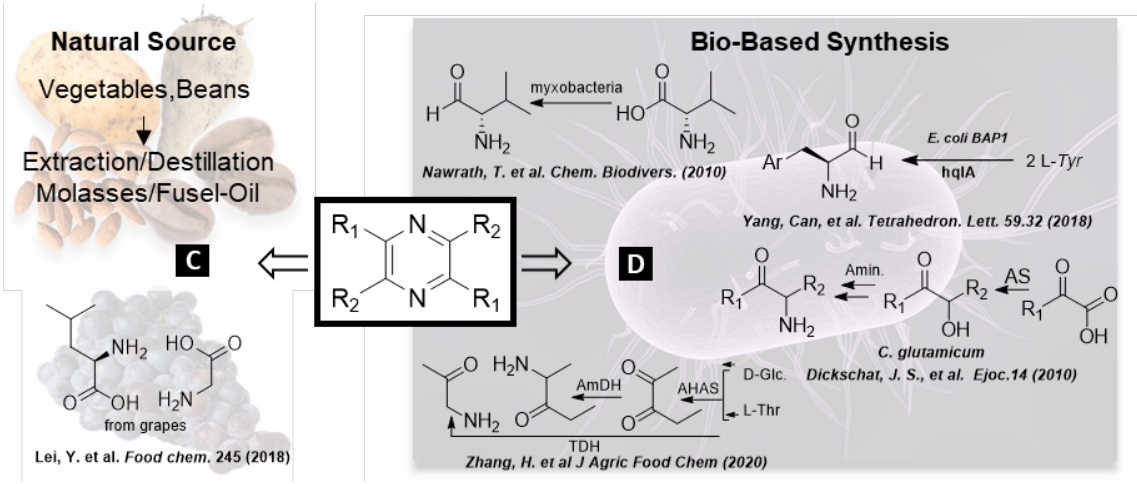

Figure 3. Retrosynthetic analysis shows different ways leading to the same molecule. Overview about different strategies: C) Extraction and distillation from natural sources (vegetables, nuts and beans) with focus on the biosynthesis of 3-isobutyl-2-methoxypyrazine in grapes from amino acids leucine and glycine and D) Biobased syntheses by in vivo biotransformation starting from amino acids and sugars.

\subsection{Chemical Synthesis}

The most prominent route for traditional pyrazine synthesis is the reaction of 1,2-diketones in the presence of ammonia to form diamines or aminoketones [22]. The list of examples is long, nevertheless an easy high yielding representative example is the reaction of diketones with diamines in methanol at room temperature 
under dry conditions in the presence of tert -BuOK in up to 5 hours, that yields above $70 \%$ of alkylated pyrazines [23].

\subsection{Enzymatic approach}

In 2018, Turner et al. reported a chemo-enzymatic synthesis of substituted pyrazines using an amino transaminase (S-selective, ATA-113, Codexis [24]) in the presence of a suitable amine donor, which mediated the key amination of the 1,2-diketone precursor to $\alpha$-aminoketones that underwent oxidative dimerization to the final product (Figure 2)[25]. In the case of pyrazines, the chirality of the amine group is irrelevant for the synthesis of the aromatic heterocycle core. All reactions were carried out at room temperature with isopropyl amine as the amine donor. Substrates were exhausted after 72 hours and pyrazine was extracted in pure form from the aqueous phase, but the yield of pyrazine was still moderate at $50-65 \%$ for symmetric11, deriving from cyclohexane-1,2-dione and $\mathbf{8}$ deriving from diacetyl, and 32\% for the non-symmetric $\mathbf{1 2}$ from pentane-2,3-dione (Figure 1). The explanation for by-product formation requires further studies e.g. identification of possible double aminated by-products in the aqueous phase or extraction problems. The question whether dimerization to the pyrazine core occurred in the aqueous buffer or in organic solvent after extraction remains elusive and needs further investigations.

On the one hand, diketones can be produced by different chemical steps from various building blocks (Figure 2 ). On the other hand, there are also biological options, since the biological route is known to be via acetoin 10 (3-hydroxy-butan-2-one, Figure 2). Recent developments have been made with a 'new' ThDP-dependent lyase, which is able to synthesize acetoin building blocks from smaller subunits e.g. pyruvate and activated acetaldehyde enzymatically. Pohl et al.showed with engineered PDC from Zymomonas mobilis ( $Z m$ PDC) and Acetobacter pasteurianus ( $A p$ PDC) they can obtain excellent yields of $61-98 \%$ from the combinations of arylated aldehydes with 1) 3-oxobutanoic acid and 2) alkyl aldehydes. At the same time, it was published how to obtain excellent pyrazine yields from either acetaldehyde with SucA [ThDP-dependent E1 subunit of the $\alpha$-ketoglutarate dehydrogenase complex from Escherichia coli(E.coli )) ], or from pyruvate (3-oxopropionic acid) with an cellobiose dehydrogenase $(C D H)[26]$.

In industrial processes, cost is one of the determining factors. The price of ATAs (e.g. ATA-113) is much higher than chemical amination with simple ammonia. The enantiomeric integrity of the amination is of great importance e.g. for pharmaceuticals; however, for the production of planar heterocycles the enantioselectivity of the amination is redundant, whereas high regioselectivity of the condensation is essential.

In this context, non-symmetric pyrazines could be synthesized regioselective with ATA- 113 [25] in an onepot approach, whereas a standard chemical synthesis would only allow access to symmetric or a mixture of non-symmetric pyrazines.

\subsection{Bio-Based Synthesis}

The production of pyrazines is abundant in plants, but so far only a few bacteria have been reported to be able to synthesize pyrazines, including Pseudomonas sp. , Bacillus sp. ,Chondromyces sp. [27], and Streptomyces sp.[28].

Furthermore, Li et al. (2017) identified the strain Paenibacillus aceti $\mathrm{L}_{1}{ }^{\mathrm{T}}$ as a highly efficient pyrazine producer by genome analysis. The strain was isolated from a solid-state acetic acid fermentation culture and is qualified for the production of $1.6 \mathrm{~g} / \mathrm{l}$ pyrazines including 2,3-di-iso -butylpyrazine, 2-iso -butyl-3methylpyrazine and 1-(5-iso -butyl-2-pyrazinyl)-1-propanone (1.6 g/l). For further understanding, the group sequenced the genome of $\mathrm{L} 14^{\mathrm{T}}$ identifying genetic structures suitable for pyrazine biosynthesis based on precursor amino acids such as valine, leucine and isoleucine as the crucial intermediates [29]. Genome analysis will form the basis for further studies on increasing selectivity and productivity using biotransformation instruments.

In nature, Corynebacterium glutamicum produces small amounts of trimethylpyrazine $\mathbf{1}$, dimethylpyrazines $\mathbf{3}, \mathbf{4 , 6}$, and tetramethylpyrazine $\mathbf{8}$, and the biosynthesis relates to the metabolism of branched-chain amino acids. Wittmannet al. (2010) have shown through a combination of gene knockout and feeding experiments 
that the biosynthetic pathway to the Top 7 (Figure 1) alkylated pyrazines, starting from pyruvate, requires the activity of acetolactate synthase (AS) and proceeds via acetolactate, followed by a sequence of transamination and oxidation steps transforming acetoin 10 to aminoketones. The last step is the condensation of aminoketones and concomitant oxidation to pyrazines [30].

In contrast, a study by Nawrath et al. (2010) highlighted a different route proposal: in Myxobacteria, pyrazines are proposed to arise from branched amino acids such as valine via reduction to valinal and dimerization of the resulting aminoaldehydes to $\mathbf{1 3}[12]$.

Silva-Junior et al. recently studied a leaf-cutter ant-associated bacterium which is producing pyrazines used as trail pheromones in ant species. The study proposed the biosynthetic pathway in the bacteriumSerratia marcescens 3B2 leading to symmetric 2,5-dimethylpyrazine $\mathbf{3}$, but more importantly the non-symmetric 3-ethyl-2,5dimethylpyrazine5a both starting with l-threonine, which is converted to aminoacetone. Subsequently, condensation and dehydration lead to compound $\mathbf{1}$. In the presence of acetate, the dehydration takes place with simultaneous addition of acetate to $\mathrm{C} 3$, resulting in a carbonyl group, which is subsequently reduced and eliminated to obtain exclusively $\mathbf{5 a}[31]$. In comparison, natural pyrazine $5 \mathbf{a}$ is only available by extraction from natural sources in mixtures with its regio-isomer 3-ethyl-2,6-dimethylpyrazine $\mathbf{5} \mathbf{b}$, which is emphasizing the importance of this selective route.

The following two examples could provide some new strategies for the enzymatic synthesis of pyrazine derivatives from natural $\alpha$-amino acids according to A) dimerization of $\alpha$-amino aldehydes and B) dimerization of $\alpha$-aminoketones in living cells.

An attempt to establish a pyrazine producing cell factory that uses the pathway via the dimerization of aldehydes was carried out in 2018 in E. coli by using a monomodular, non-ribosomal peptide synthetase HqlA from Penicillium herquei [32]. The enzyme reduces amino acids, in this case l-tyrosine, to the corresponding amino-aldehyde, which is then dimerized to the substituted pyrazines15. The binding of the acid to HqlA consumes ATP and the actual reduction requires NADPH.

Another approach was recently published (2020), in which an enzymatic cascade from Pseudomonas fluorescens SBW25 was introduced intoE. coli to produce reactive aminoketone species, which can immediately dimerize to yield pyrazines. The gene sequence included the synthesis of the natural $\alpha$-amino acid 4 -aminophenyl alanine and several other genes for the transformation to non-natural symmetric 2,5-dimethyl3,6-bis(4-aminobenzyl)pyrazine 16 . The novel biosynthetic gene cluster comprised of 4-aminophenylalanine (4APhe) C-acetyltransferase, dihydropyrazine oxidase, and a methyltransferase [33].

A potential industrial strain candidate for 2-ethyl-3,5(3,6)-dimethyl pyrazine $\mathbf{5 a / b}$ attracted the attention of Zhang et al. in 2020 after the isolation of a strain of Bacillus subtilis . They proposed the biosynthetic pathway starting from L-threonine and D-glucose, which were tested by substrate addition and isotopelabelling experiments. B. subtilis is GRAS and there is evidence for the formation of other valuable pyrazines in this organism [34].

\subsection{Industrial Applications and 'Natural' certification as driving force of bio-based approaches}

Lately, there has been an increasing call for the sustainability in industry, especially in food for daily consumption and healthy food. The global market for the flavor and fragrance industry has tripled since the beginning of the $21^{\text {st }}$ century. According to a new market research study, this industry will grow at a CAGR (Compound Annual Growth Rate) of approximately $3.1 \%$ over the next five years, reaching US\$ 30.5 billion in 2024 (US\$ 25.5 billion in 2019). In addition to the CAGR of 3.1\%, the natural and organic flavors sector is growing much faster, with an average annual growth rate of $5.7 \%$ to US $\$ 11$ billion in 2026 from US\$ 6.8 billion in 2019 [35]. This growth is mainly derived due to the global pressure for the use of flavors in food production, triggered by customer's demand as well as the increasing cost efficiency through technological progress and the concomitant possibility to declare products as natural.

The European regulation on flavors (EEC No 1334/2008) defines in article 3 (2) c): "Natural flavoring substance shall mean a flavoring substance obtained by appropriate physical, enzymatic or microbiological 
processes from material of vegetable, animal or microbiological origin either in the raw state or after processing for human consumption by one or more of the traditional food preparation processes".

To the best of our knowledge, no biotechnological syntheses for industrial production of alkylated pyrazine derivatives, with the declaration natural, have currently been registered. Thus, extraction from natural sources is the only access to natural pyrazines to this end.

\subsection{Conclusions}

In recent decades, many different bacterial and enzymatic processes for the synthesis of natural fragrances and flavors have been described in literature. Few of these processes have made it into industrial large-scale production, due to high barriers in the strongly cost-driven beverage and food industry.

In the case of alkyl pyrazines, the increasing demand can no longer be supplied economically from natural sources.

As discussed above, the consumer can no longer be satisfied by commercial chemical synthesis and the dependency on fossil oil chemicals is becoming a crucial negative factor. Production in engineered microorganisms such as Pseudomonas sp., Bacillus sp.,Chondromyces sp. and Streptomyces sp. opens possibilities for the future of small in vivo factories. Up to now, pyrazine producing strains were identified and modified by gene knockouts or transfer of genes into other organisms to produce several types of pyrazines. However, these systems are far too unspecific and produce many different products. Cell factories are more difficult to optimize compared to conventional chemistry, where typically one main product and few by-products are obtained. Recently, optimized in vivofactories of heterologous genes for pyrazine synthesis in E. colihave been thoroughly described. However, the product range has been very limited so far and has little to do with the 7 most required alkylpyrazines. For these 7 compounds, smart routes yet need to be designed. While industry will continue to improve running processes, academic developments in technological science will expand the complementary fields of chemistry and microbiology to deliver commercially affordable and sustainable products in the next decades.

\section{Acknowledgement}

C.H. has been supported by the Austrian Science Fund (FWF): [860262].

\section{Conflict of interest}

The authors declare no conflict of interest.

\section{References}

[1] a) G. Akarca, O. Tomar, İ. Güney, S. Erdur, V. Gök J. Food Sci. Technol. 2019 , 56 , 5253-5261, https://doi.org/10.1007/s13197-019-03994-1; b) S. Purkait, A. Bhattacharya, A. Bag, R. Chattopadhyay Arch. Micobiol.2020 , 1-10, https://doi.org/10.1007/s00203-020-01858-3.

[2] H. Stolterfoht, C. Rinnofner, M. Winkler, H. Pichler J. Agr. Food Chem. 2019 , 67, 13367-13392, https://doi.org/10.1021/acs.jafc.9b02690.

[3] W. Schwab, R. Davidovich-Rikanati, E. Lewinsohn Plant J.2008, 54 , 712-732, https://doi.org/10.1111/j.1365313X.2008.03446.x.

[4] a) M. Fache, B. Boutevin, S. Caillol ACS Sust. Chem. Eng.2016 , 4, 35-46, https://doi.org/10.1021/acssuschemeng.5b0134 b) A. Kundu Planta2017, 245 , 1069-1078, https://doi.org/10.1007/s00425-017-2684-x.

[5] a) N. J. Walton, M. J. Mayer, A. Narbad Phytochem.2003 , 63 , 505-515, https://doi.org/10.1016/S00319422(03)00149-3; b) G. Banerjee, P. Chattopadhyay J. Sci. Food Agric. 2019, 99 , 499-506, https://doi.org/10.1002/jsfa.9303.

[6] A. Mattia, A. Renwick, G. Sipes Food Add. Contam.2002, 135.

[7] G. Rychen, G. Aquilina, G. Azimonti, V. Bampidis, M. de Lourdes Bastos, G. Bories, P. S. Cocconcelli, G. Flachowsky, J. Gropp EFSA Journal 2017 , 15 , e04671, https://doi.org/10.2903/j.efsa.2017.4671. 
[8] A. von Wright Benef. Microorg. Agric. Food Environ.2012, 27-40.

[9] T. Brodmann, A. Endo, M. Gueimonde, G. Vinderola, W. Kneifel, W. M. de Vos, S. Salminen, C. Gómez-Gallego Front. Microbiol.2017 , 8 , 1725, https://doi.org/10.3389/fmicb.2017.01725.

[10] K. E. Murray, F. B. Whitfield J. Sci. Food Agric.1975, 26 , 973-986, https://doi.org/10.1002/jsfa.2740260714.

[11] T. Guilford, C. Nicol, M. Rothschild, B. P. Moore Biol. J. Linn. Soc. 1987, 31 , 113-128, https://doi.org/10.1111/j.10958312.1987.tb01984.x.

[12] T. Nawrath, J. S. Dickschat, B. Kunze, S. Schulz Chem. Div. 2010 , 7 , 2129-2144, https://doi.org/10.1002/cbdv.20100015

[13] B. P. Moore, W. V. Brown, M. Rothschild Chemoecol.1990 , 1 , 43-51, https://doi.org/10.1007/BF01325227.

[14] a) M. Dolezal, J. Zitko Exp. Opin. Ther. Patents2015 , 25 , 33-47, https://doi.org/10.1517/13543776.2014.982533;

b) P. B Miniyar, P. R Murumkar, P. S Patil, M. A Barmade, K. G Bothara Mini Rev. Med. Chem. 2013 , 13 , 1607-1625, DOI: 10.2174/1389557511313110007 ; c) J. A. Maga Food Rev. Int.1992 , 8 , 479-558, https://doi.org/10.1080/87559129209540951.

[15] M. Zviely Perf. Flav. 2010 , 35 , 32-34.

[16] a) D. Rowe Perf. Flav. 1998 , 23 , 9-18; b) H. C. Yeo, T. Shibamoto Trends Food Sci. Technol.1991 , 2 , 329-332, https://doi.org/10.1016/0924-2244(91)90737-4.

[17] J. A. Maga, I. Katz Crit. Rev. Food Sci. Nutr.1982, 16 , 1-48, https://doi.org/10.1080/10408398209527323.

[18] a) F. C. de Oliveira, J. S. d. R. Coimbra, E. B. de Oliveira, A. D. G. Zuñiga, E. E. G. Rojas Crit. Rev. Fodd Sci. Nutr.2016 , 56 , 1108-1125, https://doi.org/10.1080/10408398.2012.755669; b) M. Amrani-Hemaimi, C. Cerny, L. B. Fay J. Agr. Food Chem. 1995 , 43 , 2818-2822, https://doi.org/10.1021/jf00059a009; c) S. Fors, ACS Publications, 1983.

[19] M. M. Sanagi, W. P. Hung, S. M. Yasir J. Chrom. A1997, 785 , 361-367, https://doi.org/10.1016/S00219673(97)00569-4.

[20] M. P. Carter, J. P. Jensen, Google Patents, 2008 .

[21] Y. Lei, S. Xie, X. Guan, C. Song, Z. Zhang, J. Meng Food Chem. 2018 , 245 , 1141-1147, https://doi.org/10.1016/j.foodchem.2017.11.056.

[22] K. M. Klinger, F. Liebner, I. Fritz, A. Potthast, T. RosenauJ. Agr. Food Chem. 2013 , 61 , 9004-9014, https://doi.org/10.1021/jf4019596.

[23] P. Ghosh, A. Mandal Green. Chem. Lett. Rev.2012 , 5 , 127-134, https://doi.org/10.1080/17518253.2011.585182.

[24] https://www.codexis-estore.com/product-page/codex-amine-transaminase-ata-screening-kit(04/2020)

[25] J. Xu, A. P. Green, N. J. Turner Angew. Chem. Int. Ed.2018, 57 , 16760-16763, https://doi.org/10.1002/anie.201810555.

[26] M. Müller, G. A. Sprenger, M. Pohl Curr. Opin. Chem. Biol. 2013, 17, 261-270, https://doi.org/10.1016/j.cbpa.2013.02.0

[27] J. S. Dickschat, H. Reichenbach, I. Wagner-Dobler, S. SchulzEur. J. Org. Chem. 2005, 2005 , 4141-4153, https://doi.org/10.1002/ejoc.200500280.

[28] a) K. S. Rajini, P. Aparna, C. Sasikala, C. V. RamanaCrit. Rev. Mirobiol. 2011 , 37, 99-112, https://doi.org/10.3109/1040841X.2010.512267; b) A. F. Brana, M. Rodriguez, P. Pahari, J. Rohr, L. A. Garcia, G. Blanco Arch. Micobiol. 2014, 196 , 345-355, https://doi.org/10.1007/s00203-014-0977-z; c) C. A. Citron, P. Rabe, J. S. Dickschat J. Nat. Prod. 2012, 75 , 1765-1776, https://doi.org/10.1021/np300468h.

[29] P. Li, X. Gan, L. Luo, B. Du Ann. Microbiol.2017 , 67 , 391-393, https://doi.org/10.1007/s13213-0171267-1. 
[30] J. S. Dickschat, S. Wickel, C. J. Bolten, T. Nawrath, S. Schulz, C. Wittmann Eur. J. Org. Chem. 2010 ,2010 , 2687-2695, https://doi.org/10.1002/ejoc.201000155.

[31] E. A. Silva-Junior, A. C. Ruzzini, C. R. Paludo, F. S. Nascimento, C. R. Currie, J. Clardy, M. T. Pupo Sci. Rep.2018 , 8 , 1-7, https://doi.org/10.1038/s41598-018-20953-6.

[32] C. Yang, Y. Xu, K. Xu, G. Tan, X. Yu Tetrahedron Lett.2018, 59 , 3084-3087, https://doi.org/10.1016/j.tetlet.2018.06.06?

[33] S. Masuo, Y. Tsuda, T. Namai, H. Minakawa, R. Shigemoto, N. Takaya ChemBioChem 2020 , 21 , 353-359, https://doi.org/10.1002/cbic.201900448.

[34] H. Zhang, L. Zhang, X. Yu, Y. Xu J. Agr. Food Chem.2020, https://doi.org/10.1021/acs.jafc.9b07809.

[35] https://www.marketwatch.com/press-release/pyrazine-market-2019global-industry-analysis-size-share-trendsmarket-demand-growth-opportunities-and-forecast-2025-research-report-by-research-reports-world-2019-11-14. , https://www.marketwatch.com/press-release/natural-and-organic-flavors-market-2020-2026-global-size-shareemerging-trends-demand-revenue-and-forecasts-research-2020-02-14(04/2020) . 